\title{
Entropic principle and asymptotic freedom
}

\section{Citation}

Gukov, Sergei, Kirill Saraikin, and Cumrun Vafa. 2006. “Entropic Principle and Asymptotic Freedom." Physical Review D 73 (6). https://doi.org/10.1103/physrevd.73.066010.

\section{Permanent link}

http://nrs.harvard.edu/urn-3:HUL.InstRepos:41385099

\section{Terms of Use}

This article was downloaded from Harvard University's DASH repository, and is made available under the terms and conditions applicable to Other Posted Material, as set forth at http:// nrs.harvard.edu/urn-3:HUL.InstRepos:dash.current.terms-of-use\#LAA

\section{Share Your Story}

The Harvard community has made this article openly available.

Please share how this access benefits you. Submit a story.

Accessibility 
hep-th/0509109

HUTP-05/A041

ITEP-TH-55/05

\title{
The Entropic Principle and Asymptotic Freedom
}

\author{
Sergei Gukov ${ }^{1,2 \dagger}$, Kirill Saraikin ${ }^{2 \dagger}$, and Cumrun Vafa ${ }^{2}$ \\ 1 California Institute of Technology 452-48, Pasadena, CA 91125, USA \\ 2 Jefferson Physical Laboratory, Harvard University, Cambridge, MA 02138, USA
}

\begin{abstract}
Motivated by the recent developments about the Hartle-Hawking wave function associated to black holes, we formulate an entropy functional on the moduli space of Calabi-Yau compactifications. We find that the maximization of the entropy is correlated with the appearance of asymptotic freedom in the effective field theory. The points where the entropy is maximized correspond to points on the moduli which are maximal intersection points of walls of marginal stability for BPS states. We also find an intriguing link between extremizing the entropy functional and the points on the moduli space of Calabi-Yau threefolds which admit a 'quantum deformed' complex multiplication.
\end{abstract}

September 2005

$\dagger$ On leave from: ITEP, Moscow, 117259, Russia and L.D.Landau ITP, Moscow, 119334, Russia 


\section{Introduction}

There is little doubt that there exists a large number of consistent superstring vacua. This fact is not new; it has been well known for a while in the context of supersymmetric vacua. More recently, there has been some evidence that the multitude of vacua continues to exist even without supersymmetry (for introduction and references see [1],2]). Of course, one can stop here and resort to the standard philosophy of physics: Choose the theory to be in accord with observation. However, in the context of string theory, being a unified theory of all matter, it is natural to explore whether one can say a little more about the selection criteria. The purpose of this paper is to further explore one idea along these lines, advanced recently [3].

The idea in [3] is to interpret the results of [4] in the context of flux compactifications on $\mathrm{AdS}_{2} \times \mathbf{S}^{2} \times M$, where $M$ is a Calabi-Yau threefold (and where for simplicity we ignore a $\mathbb{Z}$-identification). The norm of the Hartle-Hawking wave function associated with this

background can be interpreted holographically as the black hole entropy. In particular, the flux data on the $\mathrm{AdS}_{2} \times \mathbf{S}^{2}$ geometry is mapped to the charge of the dual black hole, and the norm of the wave function satisfies

$$
\langle\psi \mid \psi\rangle=\exp (S)
$$

where $S$ denotes the entropy of the corresponding black hole. For a fixed flux data, the wave function $\psi$ can be viewed as a function over the moduli space of the Calabi-Yau, together with the choice of the normalization for the holomorphic 3-form, where the overall rescaling of the holomorphic 3 -form corresponds to the overall rescaling of the charge of the black hole. Clearly the entropy of the black hole increases as we rescale the overall charge. However, to obtain a wave function on the Calabi-Yau moduli space, one would like to get rid of this extra rescaling. The main purpose of this paper is to suggest one mechanism of how this may be done: We simply fix one of the magnetic charges, and its electric dual chemical potential. In this way, as we shall argue, the wave function becomes a function on the geometric moduli space of the Calabi-Yau and one can see which Calabi-Yau manifolds are "preferred".

It turns out that this problem can be formulated for both compact and non-compact Calabi-Yau manifolds. It is a bit more motivated in the non-compact case, since in this case there is a canonical choice of the fixed charge (D0 brane in the type IIA context). We find that the condition for a maximum/minimum corresponds to the points of intersection 
of walls of marginal stability. Moreover we find a set of solutions in various examples. One type of solutions we find corresponds to points on moduli space which admit a complex multiplication type structure. We also find examples where extrema correspond to the appearance of extra massless particles. We find that in these cases the norm of the wave function $\psi$ is maximized in correlation with the sign of beta function: Asymptotically free theories yield maximum norm for the wave function.

The organization of this paper is as follows: we start in section 2 with the formulation of the problem. Then, in section 3, we find the conditions for maxima/minima of the wave function. We also explain why this favors asymptotically free theories in local examples where there are massless fields. In section 4 we give examples of our results and in section 5 we end with conclusions and some open questions.

\section{General Formulation of the Problem}

Consider compactifications of type IIB superstrings on $\mathrm{AdS}_{2} \times \mathbf{S}^{2} \times M$ where $M$ is a Calabi-Yau threefold, which may or may not be compact. In addition we consider fluxes of the 4-form gauge field along $\mathrm{AdS}_{2} \times \mathbf{S}^{2}$ and 3-cycles of $M$. Let $F_{p, q}=p^{I} \alpha_{I}+q_{J} \beta^{J}$ denote the flux through $M$, where $\alpha_{I}$ and $\beta^{J}$ form a canonical symplectic basis for integral 3-form cohomology $H^{3}(M, \mathbb{Z})$. According to [3], the results of [4] can be interpreted in terms of a Hartle-Hawking type wave function $\psi_{p, q}$ for this geometry on the minisuperspace, with the property that

$$
\left\langle\psi_{p, q} \mid \psi_{p, q}\right\rangle=\exp S(p, q)
$$

Here $S(p, q)$ denotes the entropy of the dual black hole obtained by wrapping a D3 brane with magnetic and electric charges $p$ and $q$.

In the limit of large fluxes,

$$
(p, q) \rightarrow \lambda(p, q)
$$

where $\lambda>>1$, the entropy $S(p, q)$ has a classical approximation, given by the BekensteinHawking formula. In this limit, the curvature of $\mathrm{AdS}_{2} \times \mathbf{S}^{2}$ becomes small, and the entropy of the black hole is equal to $\frac{1}{4} A\left(\mathbf{S}^{2}\right)$. In particular, the attractor mechanism [5] will freeze the complex structure moduli of the Calabi-Yau space $M$, so that there exists a holomorphic 3 -form $\Omega$ on $M$ with the property

$$
\operatorname{Re}(\Omega)=F_{p, q}
$$


Moreover, in this limit the entropy is given by

$$
S(p, q)=\frac{A\left(\mathbf{S}^{2}\right)}{4}=-i \frac{\pi}{4} \int_{M} \Omega \wedge \bar{\Omega}
$$

where $\Omega$ fixed by (2.3). Furthermore, in this limit, $\operatorname{Im} \Omega$ plays the role of the chemical potential.

Suppose we wish to ask the following question: In type IIB compactification on $\mathbb{R}^{4} \times$ $M$, which Calabi-Yau $M$ is "preferred"? One way to tackle this question is to embed it in the geometry $\mathrm{AdS}_{2} \times \mathbf{S}^{2} \times M$ where the complex structure of $M$ is determined by the fluxes, through the attractor mechanism. Then, the question becomes: For which values of the complex structure moduli the norm of the wave function is maximized or, in other words, for which attractor Calabi-Yau the entropy of the corresponding black hole is maximized? In this formulation of the question, we can view $\mathbb{R}^{4}$ as a special limit of $\mathrm{AdS}_{2} \times \mathbf{S}^{2}$ where the charge of the black hole is rescaled by an infinite amount $\lambda \rightarrow \infty$. Thus $\mathrm{AdS}_{2} \times \mathbf{S}^{2}$ can be viewed as a regulator geometry for $\mathbb{R}^{4}$.

However, this way of asking the question leads to the following pathology: The entropy of the black hole for large $\lambda$ scales as $\lambda^{2}$. Therefore, in order to get a reasonable function on the moduli space of $M$ we need to fix the normalization of $\Omega$. One way to do this is to fix the value of $\int \Omega \wedge \bar{\Omega}$ so that it is the same at all points in moduli; however this is precisely the entropy that we wish to maximize. If we fixed the normalization of $\Omega$ in this way, we would obtain, tautologically, a flat distribution on the moduli space of $M$. In this sense there would be no particular preference of one point on the moduli of $M$ over any other. Instead we consider the following mathematically natural alternative: We choose a 3-cycle $A_{0} \subset M$ and fix the normalization of $\Omega$ by requiring it to have a fixed period along $A_{0}$

$$
\int_{A_{0}} \Omega=\text { fixed }
$$

Since the overall scale of $\Omega$ does not affect the extremum point on the moduli space of $M$, with no loss of generality we can fix the above period to be 1 . Then, we can consider maximizing the entropy, which now depends only on the geometric moduli of $M$. Thus our problem becomes

$$
\text { Maximize }\left|\int \Omega \wedge \bar{\Omega}\right| \quad \text { subject to } \quad \int_{A_{0}} \Omega=1 \text {. }
$$


Physically, what this means is that we fix one of the charges of the black hole, say the magnetic charge $p^{0}=1$, and the corresponding electric chemical potential $\phi^{0}=0$ (determined by the imaginary part of $\Omega$ ).

Strictly speaking, the above problem is well defined on the moduli space of CalabiYau manifolds together with a choice of a 3-cycle. This, in general, is a covering of the moduli space. Nevertheless, any particular maximization of the entropy functional on this covering space will descend to a particular choice of the complex structure moduli of $M$ (by forgetting on which sheet the function is maximized). Of course, it would be interesting to find out whether or not this covering of the moduli space is a finite covering or not. We will discuss some aspects of this in section 5. We should point out, however, that there is a canonical choice of the cycle $A_{0}$ in the mirror type IIA problem, where one is studying even-dimensional D-branes wrapped over even-dimensional cycles of a noncompact Calabi-Yau manifold. In this case, one can choose $A_{0}$ to be a point on $M$ and consider a fixed number of $D 6$ branes with zero chemical potential for D0 branes. We also note that, in the type IIA setup, fixing one of the periods can be interpreted as fixing the topological string coupling constant

$$
X^{0}=\frac{4 \pi i}{g_{s}}
$$

In what follows, we consider both compact and non-compact examples.

\section{General Conditions for Maxima/Minima}

In this section, we derive equations for the critical points of the constrained variational problem described in the previous section, and discuss maxima of the entropy functional

$$
S=-i \frac{\pi}{4} \int_{M} \Omega \wedge \bar{\Omega}
$$

It turns out that the critical points of (3.1) are described by equations of the form

$$
\operatorname{Im} a^{D}-\tau \operatorname{Im} a=0,
$$

where $\left(a, a^{D}\right)$ denote "reduced" periods of the Calabi-Yau and $\tau=\frac{d a^{D}}{d a}$ is the coupling constant matrix. The points on the moduli space where the entropy is maximized are those where $\operatorname{Im} \tau>0$ and all but one of the Calabi-Yau periods have equal phase. As we explain below, these are also the points where the maximal number of the walls of marginal stability for BPS states meet together. Notice, the restriction $\operatorname{Im} \tau>0$ implies that the effective field theory for the extra massless particles appearing at the maximum point can be decoupled from the gravity. This is a necessary condition for the asymptotically free effective theories. 


\subsection{Critical Points}

In order to extremize the functional (3.1) we need to introduce coordinates on the moduli space of a Calabi-Yau manifold with one fixed 3-cycle. Given the symplectic basis of 3-cycles $\left\{A_{I}, B^{J}\right\}_{I, J=0, \ldots, h^{2,1}}$, such that $\#\left(A_{I}, B^{J}\right)=\delta_{I}^{J}$, the periods of the holomorphic 3-form are

$$
\begin{aligned}
& X^{I}=\int_{A_{I}} \Omega \\
& F_{I}=\int_{B^{I}} \Omega
\end{aligned}
$$

In particular, one can use $X^{I}$ as the projective coordinates on the Calabi-Yau moduli space, and express the $B$-periods as derivatives of the prepotential:

$$
F_{I}(X)=\frac{\partial \mathcal{F}_{0}(X)}{\partial X^{I}}
$$

We choose the fixed 3 -cycle to be $A_{0}$. Then the normalization of $\Omega$ is fixed by the condition

$$
X^{0}=\int_{A_{0}} \Omega
$$

As we discussed earlier, we can always set $X^{0}=1$. However, in what follows it will be useful to keep the dependence on $X^{0}$ which, in the type IIA context, determines the topological string coupling constant, $c f$. (2.6).

It is natural to use the following coordinates on the moduli space of Calabi-Yau manifolds with a fixed 3-cycle:

$$
a^{i}=\frac{X^{i}}{X^{0}}, \quad i=1, \ldots, h^{2,1}
$$

Also, we introduce the "dual" variables

$$
a_{i}^{D}=\frac{F_{i}}{X^{0}}
$$

and a "rigid" prepotential

$$
F(a)=\left(X^{0}\right)^{-2} \mathcal{F}_{0}(X(a))
$$

Then, using the fact that $\mathcal{F}_{0}$ is a homogeneous holomorphic function of degree two, we find

$$
F_{0}=X^{0}\left(2 F-a^{i} a_{i}^{D}\right)
$$


Therefore, the functional (3.1) can be written as

$$
S=i \frac{\pi}{4}\left|X^{0}\right|^{2}\left\{2(F-\bar{F})-\left(a^{i}-\bar{a}^{i}\right)\left(a_{i}^{D}+\bar{a}_{i}^{D}\right)\right\}
$$

This is, of course, the standard expression for the Kähler potential $S=\frac{\pi}{4} e^{-K}$, written in terms of the special coordinates, see e.g. [6].

Extremizing the action $(\underline{3.10})$ with respect to $a^{i}$ and $\bar{a}^{i}$, we obtain the following system of equations:

$$
\operatorname{Im} a_{i}^{D}-\tau_{i j} \operatorname{Im} a^{j}=0
$$

where the coupling constant matrix $\tau_{i j}$ is given by

$$
\tau_{i j}=\frac{\partial^{2} F}{\partial a^{i} \partial a^{j}} \equiv \frac{\partial^{2} \mathcal{F}_{0}}{\partial X^{i} \partial X^{j}}
$$

Solutions to the equations (3.11) define critical points on the Calabi-Yau moduli space. Our goal will be to study these points and to understand their physical and/or geometric meaning.

Before we proceed, let us make a few general comments about the form of the equations (3.11). First, note that (3.11) is a system of non-linear complex equations. Even though these equations are not differential, finding their solutions is a challenging and interesting problem. To see this, let us write (3.11) in the following form

$$
\operatorname{Im} \partial_{i} F-\left(\partial_{i} \partial_{j} F\right) \operatorname{Im} a^{j}=0
$$

where we expressed $a_{i}^{D}$ and $\tau_{i j}$ in terms of the single function $F\left(a^{i}\right)$. For a given CalabiYau space $M$ and a choice of the 3-cycle, the function $F\left(a^{i}\right)$ is fixed; it is generically a non-trivial transcendental function. Therefore, (3.11) (equivalently (3.13)) represents a system of $n=h^{2,1}$ complex equations for $n$ complex variables $a^{i}, i=1, \ldots, n$. Therefore one expects that solutions to these equations are isolated points in the moduli space.

Let us note that Calabi-Yau manifolds which correspond to these points admit special structures, analogous to the complex multiplication. The notion of complex multiplication for higher dimensional varieties goes back to the work of Mumford [7]; in the context of Calabi-Yau three-folds it was studied by Borcea [8]. In the physics literature, it appears

1 Here we used the Riemann bilinear identity $\int_{M} \alpha \wedge \beta=\sum_{I}\left(\int_{A_{I}} \alpha \int_{B^{I}} \beta-\int_{A_{I}} \beta \int_{B^{I}} \alpha\right)$. 
in the study of black hole attractors [9] and rational conformal field theories [10]. Let us recall the attractor equations [5]

$$
\begin{aligned}
2 \operatorname{Im} a^{i} & =p^{i} \\
2 \operatorname{Im} a_{i}^{D} & =q_{i}
\end{aligned}
$$

where $(p, q) \in \mathbb{Z}^{n}$ denote magnetic and electric fluxes. One says that a Calabi-Yau manifold admits complex multiplication if the Jacobian $T=\mathbb{C}^{n} /\left(\mathbb{Z}^{n}+\tau \mathbb{Z}^{n}\right)$ associated with the coupling constant matrix $\tau_{i j}$ admits complex multiplication. This occurs if $\tau_{i j}$ satisfies the following second order matrix equation:

$$
\tau C \tau+A \tau-\tau D-B=0
$$

where $A, B, C, D$ are some integer matrices. It is straightforward to check that this is indeed the case for a suitable choice of the integer matrices2. Therefore, any Calabi-Yau with moduli fixed by the attractor mechanism (3.14) and satisfying equation (3.11) admits complex multiplication. Notice, that Jacobian $T$ for this Calabi-Yau is singular, since (3.11) implies that there are fixed points under the $\mathbb{Z}^{n}+\tau \mathbb{Z}^{n}$ action.

Solutions to (3.11) fall into three families, which can be characterized by the imaginary part of the coupling constant matrix $\tau_{i j}$. To see this, notice that the imaginary part of extremum equations (3.11) is given by

$$
\operatorname{Im} \tau_{i j} \cdot \operatorname{Im} a^{j}=0
$$

Therefore, if $\operatorname{Im} \tau_{i j}$ is non-degenerate (that is if $\operatorname{det}|| \operatorname{Im} \tau_{i j} \| \neq 0$ ), the only possible solution is $\operatorname{Im} a^{i}=0$. Moreover, assuming that $\tau_{i j}$ remains finitel, it also follows that $\operatorname{Im} a_{i}^{D}=0$. We shall refer to this family of solutions as solutions of type I:

$$
\operatorname{Im} a^{i}=0, \quad \operatorname{Im} a_{i}^{D}=0, \quad i=1, \ldots, n
$$

The expression for the entropy functional calculated at the critical point of type I turns out to be very simple:

$$
S_{*}=i \frac{\pi}{2}\left|X^{0}\right|^{2}(F-\bar{F})
$$

If we go to the conventional topological string notations

$$
F_{\text {top }}=i(2 \pi)^{3} F, \quad Z=\exp \frac{1}{g_{s}^{2}} F_{t o p}
$$

\footnotetext{
2 For example, $A=n \vec{q} \otimes \vec{q}, \quad B=0, \quad C=m \vec{p} \otimes \vec{p}-n \vec{p} \otimes \vec{q}, \quad D=m \vec{p} \otimes \vec{q}$

3 or if $\tau \sim a^{-\alpha}$, where $\alpha<1$
} 
and use (2.6), we see that the probability function for the Calabi-Yau at the critical point is given by the square of the topological wave function $\Psi_{t o p}=Z$, in accordance with [3, 4 :

$$
e^{S_{*}}=\left|\Psi_{t o p}\right|^{2}
$$

Solutions of type II correspond to $\operatorname{det}|| \operatorname{Im} \tau_{i j} \|=0$. They can be expressed in terms of real eigenvectors $v^{i}$ of the coupling constant matrix, $\operatorname{Im} \tau_{i j} \cdot v^{j}=0$,

$$
\operatorname{Im} a^{i}=v^{i}, \quad \operatorname{Im} a_{i}^{D}=\tau_{i j} v^{i}, \quad i, j=1, \ldots, n
$$

Finally, solutions of type III correspond to divergent coupling constant matrix:

$$
\operatorname{Im} a^{i}=0, \quad \operatorname{Im} a_{i}^{D}=\lim _{\operatorname{Im} a^{i} \rightarrow 0} \operatorname{Re} \tau_{i j} \cdot \operatorname{Im} a^{i} \neq 0, \quad i=1, \ldots, n
$$

All types of the solutions represent some special points on the Calabi-Yau moduli space.

\subsection{Conditions for Maximum/Minimum}

It is natural to ask which of these critical points are maxima and which are minima. The former correspond to theories which are preferred, while the latter correspond to the theories which are least likely, according to the entropic principle. Therefore, our main goal is to search for the maximum points.

In order to answer this question, we need to look at the second variation of the action at the critical point:

$$
\delta^{2} S=-\frac{\pi}{2}\left|X^{0}\right|^{2}\left(\operatorname{Im} \tau_{i j}\right) \delta a^{i} \delta \bar{a}^{j}+\frac{\pi}{4}\left|X^{0}\right|^{2} \operatorname{Im} a^{i}\left(c_{i j k} \delta a^{j} \delta a^{k}+\bar{c}_{i j k} \delta \bar{a}^{j} \delta \bar{a}^{k}\right)
$$

where $c_{i j k}$ are defined as

$$
c_{i j k}=\frac{\partial^{3} F}{\partial a^{i} \partial a^{j} \partial a^{k}}
$$

The bilinear form $\operatorname{Im} a^{i} c_{i j k} \delta a^{j} \delta a^{k}$ does not have a definite signature. This means that if it is non-zero, the critical point is neither minimum nor maximum. Therefore, a necessary condition for the critical point to be a local maximum is

$$
c_{i j k} \operatorname{Im} a^{k}=0
$$


Let us concentrate on the first term in (3.23), assuming that this condition is satisfied. Remember that reduced coupling constant matrix $\tau_{i j}$ is part of a full matrix $\tau_{I J}$. Imaginary part of this matrix has signature 1 of type $\left(h^{2,1}, 1\right)$, as follows from the expression

$$
\operatorname{Im} \tau_{I J}=\frac{i}{2} \int \partial_{I} \Omega \wedge \bar{\partial}_{J} \bar{\Omega}
$$

and decomposition $\partial_{I} \Omega \in H^{2,1}(M) \oplus H^{3,0}(M)$. Therefore, the signature of the reduced matrix $\tau_{i j}$ is either $\left(h^{2,1}, 0\right)$ or $\left(h^{2,1}-1,1\right)$. In the first case the form $\left(\operatorname{Im} \tau_{i j}\right) \delta a^{i} \delta \bar{a}^{j}$ is positive definite and therefore, the entropy functional has

$$
\text { maximum, if } \operatorname{Im} \tau_{i j}>0
$$

In the second case generically we have a saddle point, and a minimum if $h^{2,1}=1$. Thus we conclude that, in general, Calabi-Yau models with $\operatorname{Im} \tau_{i j}>0$ are preferred.

The extremum conditions (3.11) are very restrictive, but it is hard to find a solution in general case. However, if we want to satisfy constraint (3.25), which is necessary for maximization of the entropy, the problem simplifies. Assuming that $c_{i j k}$ is not of special degenerate form, a general solution to this constraint is $\operatorname{Im} a^{i}=0$, and therefore we should look at the type I solution

$$
\operatorname{Im} a^{i}=\operatorname{Im} a_{i}^{D}=0
$$

Physically, this is a particularly natural choice and we can explain it in yet another way: The extremum of the probability density given by the entropy functional, should naturally pick attractor fixed points. In our problem the electric chemical potential is set to zero and the magnetic charge is fixed in one particular direction. It is not surprising that this means that the rest of the charges are set to zero at the extremum. In particular the equation (3.28) can naturally be interpreted as the attractor point with this set of charges.

Another way to derive 3.28 is to notice that imaginary part of the coupling constant matrix enters the second variation of the action (3.23) and therefore it should be nondegenerate at the local minimum or maximum point:

$$
\operatorname{det}|| \operatorname{Im} \tau_{i j} \| \neq 0
$$

4 The simplest illustration is a toy model with the cubic prepotential, $\mathcal{F}_{0}=-\frac{1}{3} \frac{\left(X^{1}\right)^{3}}{X^{0}}$. It is easy to check that the signature of $\operatorname{Im} \tau_{I J}$ in this example is $(1,1)$. Notice, that there is a difference between the physical coupling constant matrix, including the graviphoton coupling, and $\tau_{I J}$. The physical coupling constant matrix is always positive definite (see [1]). 
Combining this with the imaginary part of extremum equations (3.16), we get $\operatorname{Im} a^{i}=0$.

One of the interesting examples of the maximum entropy solutions is the one with the logarithmic behavior of the coupling constant matrix (explicit examples of this are discussed in the next section):

$$
\tau=\tau_{0}+i \beta \log \frac{a^{2}}{\Lambda^{2}}
$$

near the critical point $a=0$. From the point of view of the corresponding effective field theory in four dimensions this expression describes an RG flow of the couplings, and $\beta$ is the one-loop beta function of the effective field theory near the point $a=0$. Combining this with the maximum condition $\operatorname{Im} \tau_{i j}>0$ discussed earlier, we conclude that for the theories with $\beta<0$, that is, for asymptotically free theories the probability density is maximized.

\subsection{Marginal Stability Curves and the Entropy}

As we will explain below, the conditions (3.28) imply that the points where the entropy is maximized correspond to points on the moduli space which are maximal intersection points of the marginal stability walls for BPS states. The solutions to (3.28) can be characterized in purely geometric terms. Let us consider the type IIB setup and look at the periods (3.2) - (3.3) of the Calabi-Yau. Since we used the gauge $X^{0}=1$, the condition (3.17) means that exactly $2 h^{2,1}+1$ periods, namely $\left(X^{0}, X^{i} ; F_{i}\right)$ are real. However, the last period $F_{0}=\frac{2}{X^{0}} \mathcal{F}_{0}-\frac{X^{i}}{X^{0}} F_{i}$ does not have to be real, since the phase of the prepotential is not fixed by the phase of its derivatives. In fact, if all of the periods were real, the holomorphic volume of the Calabi-Yau $\int \Omega \wedge \bar{\Omega}=\left(X^{I} \bar{F}_{I}-\bar{X}^{I} F_{I}\right)$ would be zero. Considering such singular Calabi-Yau would hardly make sense, as it implies that $\Omega$ is pointwise zero on the Calabi-Yau. Fortunately, this is not the case since $F_{0}$ is not real. Thus the geometry of periods is as given in Figure 1.

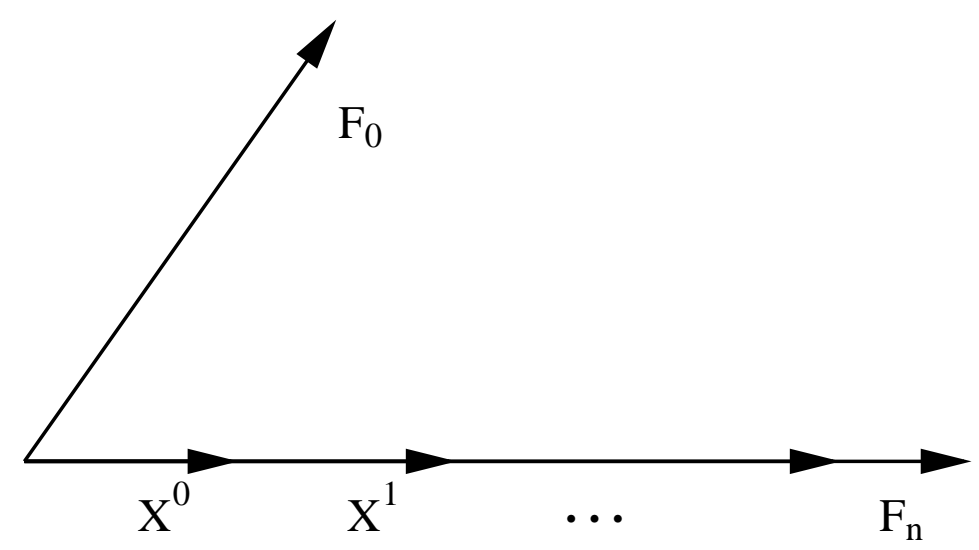

Fig. 1: Calabi-Yau periods at the maximum entropy point on the moduli space. 
Note that a point where all periods but one are aligned is a point where a maximal number of walls of marginal stability for BPS states meet together. In fact, this is the strongest condition we can have; generically, it is impossible to have all periods aligned as the number of constraints would be one higher than the number of parameters. So, the condition for a maximum for the probability is the same as maximal marginality for BPS bound states.

One can actually relax the condition $X^{0}=1$, and introduce an arbitrary phase $X^{0}=$ $e^{i \phi}$ instead. This will accordingly rotate all other periods, resulting in an equivalent CalabiYau manifold. Therefore, we can formulate an alternative maximum criterion: The entropy functional is maximized for the points on the moduli space where all but one of the CalabiYau periods are aligned on the complex plane, and $\operatorname{Im} \tau_{i j}>0$.

Suppose now that we can find such a point, where exactly $\left(2 h^{2,1}+1\right)$ of the periods are aligned. We should stress that for a given prepotential these aligned periods can actually be some linear combinations of the canonical $A$ and $B$ periods (3.2)-(3.3). Is there a freedom to choose, which of them we should use to fix the normalization of $\Omega$ in the maximization problem (2.5), or there is a canonical choice of the cycle $A_{0}$ ? The answer to the last question is positive: the cycle $A_{0}$ is dual to the 3-cycle which is not aligned with the rest of the 3 -cycles. In other words, $A_{0}$ corresponds to the null vector in the space of $\left(2 h^{2,1}+1\right)$ aligned cycles with respect to the intersection pairing. Thus, given the point on the moduli space where all but one of the Calabi-Yau periods are aligned, the cycle $A_{0}$ is determined uniquely. In the next section, we will illustrate this with a simple example of the quintic three-fold.

Similarly, in the type IIA setup, we can consider bound states of D0, D2, and D4 branes with charges $n_{0}, \vec{n}_{2}$, and $\vec{n}_{4}$, respectively. The BPS mass of such states is given by the standard formula

$$
M_{B P S}=|Z|=\left|n_{0}+\vec{n}_{2} \cdot \vec{a}+\vec{n}_{4} \cdot \vec{a}^{D}\right|
$$

Since in our case $\operatorname{Im} a_{i}=\operatorname{Im} a_{i}^{D}=0$, it follows from the BPS formula (3.31) that the critical points of type I are precisely the points in the moduli space, where all bound states of D0, D2, and D4 branes become marginal,

$$
M_{B P S}\left(n_{0}, \vec{n}_{2}, \vec{n}_{4}\right)=M_{B P S}\left(n_{0}, 0,0\right)+M_{B P S}\left(0, \vec{n}_{2}, 0\right)+M_{B P S}\left(0,0, \vec{n}_{4}\right)
$$

\section{Examples}

In this section we will discuss two types of examples, corresponding to non-compact and compact Calabi-Yau cases. There is a crucial difference between these two cases in 
the type IIA setup. Namely, on a compact Calabi-Yau manifold $M$, the cycles undergo monodromies as one goes around singularities in the moduli space, while on a non-compact Calabi-Yau there always exists at least one cycle (0-cycle in the type IIA frame) which does not undergo monodromy. Therefore, this is a canonical cycle to fix the period. However, as we will see, the general approach based on (2.5) works in both cases.

As we discussed in the previous section, the problem of finding the maximum points on the moduli space is equivalent to the problem of finding the points where all but one of the Calabi-Yau periods are aligned. Unfortunately, at the moment it is unknown how to find all such points for a given class of Calabi-Yau manifolds. Our approach below is to look at the well known special points on the moduli space (singularities, large complex structure, etc.) as potential candidates. Therefore, our list of examples is far from complete and serves just as an illustration of the general idea. We find two types of solutions: Solutions which correspond to points on Calabi-Yau moduli which admit a structure similar to complex multiplication. The other type of solutions corresponds to points on the moduli space where we have massless particles. In the non-compact case the only set of examples where we actually find a maximum, as opposed to minimum or other extremum points, is when we have extra massless fields which lead to an asymptotically free gauge theory.

\subsection{The Local $\mathbf{C P}^{1}$}

Let us start with the two simplest local models for a non-compact Calabi-Yau manifolds, the total space of $\mathcal{O}(-1) \oplus \mathcal{O}(-1) \rightarrow \mathbf{C P}^{1}$ (conifold singularity), and the total space of $\mathcal{O}(0) \oplus \mathcal{O}(-2) \rightarrow \mathbf{C P}^{1}$. (The exact stringy wave function for the conifold model with an infinite set of non-normalizable deformations was studied in [12].) It is instructive to look first at the infinite product representation of the topological string partition function on the A-model side [13]. For the conifold we haves]:

$$
Z_{(-1,-1)}=\frac{\prod_{n=1}^{\infty}\left(1-q^{n} Q\right)^{n}}{\prod_{n=1}^{\infty}\left(1-q^{n}\right)^{n}}
$$

5 Here we use topological string conventions: $t=2 \pi(J-i B)$. Sometimes an alternative convention $t=B+i J$ is used in the literature, since then the mirror map is given by $t=\frac{X^{1}}{X^{0}}$. In notations (3.6) then we have $a=B+i J$. 
where $q=e^{-g_{s}}$ and $Q=e^{-t}$, while for $\mathcal{O}(0) \oplus \mathcal{O}(-2) \rightarrow \mathbf{C P}^{1}$ case:

$$
Z_{(0,-2)}=\frac{\prod_{n=1}^{\infty}\left(1-q^{n}\right)^{n}}{\prod_{n=1}^{\infty}\left(1-q^{n} Q\right)^{n}}
$$

In the semiclassical limit $q \rightarrow 1$, which is the limit we are most interested in, the above expressions depend on $Q$ only. Moreover, the first expression decreases while the second increases rapidly as $Q \rightarrow 1$. And as we will see in a moment, the entropy functional has a minimum for the conifold, and a maximum for $\mathcal{O}(0) \oplus \mathcal{O}(-2) \rightarrow \mathbf{C P}^{1}$ at $t=0$. Indeed, since $Z=\exp \left(\frac{1}{g_{s}^{2}} F_{t o p}\right)$, the entropy functional (3.10) in this representation is given by

$$
e^{S}=|Z|^{2} e^{-\frac{1}{2}(t+\bar{t})\left(\frac{\partial}{\partial t} \log Z+\frac{\partial}{\partial \bar{t}} \log \bar{Z}\right)}
$$

As expected, the second variation of the functional at the extremum is equal to

$$
\frac{\delta^{2} S}{\delta t \delta \bar{t}}=-\frac{2 \pi}{g_{s}^{2}} \operatorname{Im} \tau
$$

where

$$
\tau=i \frac{g_{s}^{2}}{2 \pi} \frac{\partial^{2}}{\partial t^{2}} \log Z
$$

At the conifold point we have $t=t^{D}=0$. Therefore 3.17) is satisfied and the conifold point is an extremum. Moreover, near $t=0$ we have

$$
\tau=\frac{i}{2 \pi} \log t+\ldots
$$

Hence, $\operatorname{Im} \tau<0$, which means that the conifold point is a minimum. On the contrary, since the infinite product expression (4.2) for the total space of $\mathcal{O}(0) \oplus \mathcal{O}(-2) \rightarrow \mathbf{C P}^{1}$ is given by the inverse of (4.1), in this case we have $\operatorname{Im} \tau>0$ at $t=0$. Therefore, this is a maximum point.

One can arrive to the same conclusion by looking at the genus-zero prepotential, which is equivalent to the approach based on the infinite-product formula (4.1). For example, for the conifold, we have

$$
F_{\text {top }}^{0}=\frac{1}{12} t^{3}-\frac{\pi^{2}}{6} t+\zeta(3)-\sum_{n=0}^{\infty} \frac{e^{-n t}}{n^{3}}
$$

and therefore 


$$
\tau=\frac{i}{2 \pi} \log \left(1-e^{-t}\right)+\ldots
$$

in agreement with (4.6). For the total space of $\mathcal{O}(0) \oplus \mathcal{O}(-2) \rightarrow \mathbf{C P}^{1}$ we get the same expression with an extra minus sign.

The difference between these two examples is very instructive: At the conifold point, where the entropy in minimized, we have extra massless hypermultiplet, while at $t=0$ locus of $\mathcal{O}(0) \oplus \mathcal{O}(-2) \rightarrow \mathbf{C P}^{1}$, where the entropy in maximized, an extra massless vector multiplet appears. The morale of the story is that Calabi-Yau manifold providing the vector multiplet is preferred.

Let us conclude with a few general remarks. Suppose we use topological strings to obtain information about the effective supersymmetric four-dimensional gauge theories with matter fields and interactions which, in principle, can have applications to phenomenological models of particle physics. This can be done, for example, in the context of the geometrical engineering program in string theory. Here, topological strings provide a nice laboratory, since many exact results for the topological string partition function are available.

In the example we just studied, the distinction between these two cases directly corresponds to whether or not the effective field theory is asymptotically free. In particular, the appearance of a vector multiplet, which corresponds to an asymptotically free theory, is preferred over the non-asymptotically free theory, where we have an extra massless hypermultiplet. From the effective field theory viewpoint, this is translated into the statement that asymptotically free effective theories are preferred. One can also study other special points in moduli space, such as points which correspond to conformal fixed points. In general, these will involve more moduli parameters, and one might expect that in these cases we will have a mixture of the above results: along some directions the probability for conformal fixed point is maximized and along others it is minimized. In other words, it is an extremum for the entropy functional, but not a definite maximum or minimum. In fact we will discuss an explicit example which corresponds to a multi-parameter model where one finds that it is an extremum but not a pure maximum or a minimum (see sec. 4.4 below). 


\subsection{Large Complex Structure Limit}

Below we will argue that near the large complex structure limit in the one-parameter Calabi-Yau models there is an infinite family of solutions to the extremum equations (3.11), labeled by an integer number. Namely, for the values of the complexified Kähler parameter $t$, such that $\operatorname{Re} t=0$ and $\operatorname{Im} t \gg 1$, there are infinitely many points where exactly three of the periods are aligned. In a sense, these are the points where the Calabi-Yau admits a deformed complex multiplication. Indeed, at these points, to the leading approximation, $\tau$ satisfies a quadratic equation with integer coefficients. The entropy functional (3.1) has a maximum for all such points.

Let us start with a simple model with the cubic prepotential

$$
\mathcal{F}_{0}=-\frac{1}{3} \frac{\left(X^{1}\right)^{3}}{X^{0}}
$$

This model captures the leading behavior of all one-parameter Calabi-Yau models in the large complex structure limit. It can also be viewed as the exact prepotential for some parts of the CY moduli which receive no quantum corrections (such as the Kähler moduli of $T^{2}$ 's inside CY threefolds). The vector of periods is given by

$$
\left(\begin{array}{c}
X^{0} \\
X^{1} \\
F_{1} \\
F_{0}
\end{array}\right)=\left(\begin{array}{c}
X^{0} \\
X^{1} \\
-\left(X^{1}\right)^{2} / X^{0} \\
\frac{1}{3}\left(X^{1}\right)^{3} /\left(X^{0}\right)^{2}
\end{array}\right)
$$

Let us consider these periods on the subspace $\operatorname{Im} X^{0}=0$ and $\operatorname{Re} X^{1}=0$. In other words, let us set the $B$-field to zerol. Then, two of the periods (namely, $X^{1}$ and $F_{0}$ ) are purely imaginary, and two $\left(X^{0}\right.$ and $\left.F_{1}\right)$ are real. However, at the points where

$$
\left(\frac{X^{1}}{X^{0}}\right)^{2}=n
$$

for some (negative) integer $n$, the linear combination of the periods $F^{1}+n X^{0}$ vanishes! This means that at these points the following three periods are aligned: $\left(X^{1}, F_{0}, F^{1}+n X^{0}\right)$; they take purely imaginary values, while $X_{0}$ is real. According to our general principle, these points are extremum points of the entropy functional. The main question now is whether this extremum is a minimum or a maximum.

${ }^{6}$ We use conventions, where $t=a=\frac{X^{1}}{X^{0}}=B+i J$. 
In order to answer this question we need to identify the 3 -cycle $A_{0}$, to find the resulting prepotential, and to calculate the imaginary part of the coupling constant. We use $S L(4, \mathbb{Z})$ transformation to bring the periods into the following form

$$
\left(\begin{array}{c}
X^{0} \\
X^{1} \\
F_{1}+n X^{0} \\
F_{0}+n X^{1}
\end{array}\right)=\left(\begin{array}{cccc}
1 & 0 & 0 & 0 \\
0 & 1 & 0 & 0 \\
n & 0 & 1 & 0 \\
0 & n & 0 & 1
\end{array}\right)\left(\begin{array}{c}
X^{0} \\
X^{1} \\
F_{1} \\
F_{0}
\end{array}\right)
$$

As we discussed in section 3.3, the cycle $A_{0}$ corresponds to the null direction in the space spanned by the aligned periods. In the present case, it is easy to see that the period corresponding to this 3 -cycle is $F_{0}+n X^{1}$. Therefore, normalizing the value of the period of $\Omega$ over this 3-cycle to unity, as in (2.5), is equivalent to rescaling of all the periods by $\left(F_{0}+n X^{1}\right)^{-1}$. Then, the period vector takes the form:

$$
\left(\begin{array}{c}
-2 \widetilde{\mathcal{F}}_{0}+\widetilde{X}^{1} \widetilde{F}_{1} \\
-\widetilde{F}_{1} \\
\widetilde{X}^{1} \\
1
\end{array}\right)=\left(\begin{array}{c}
\frac{1}{n t+t^{3} / 3} \\
\frac{t}{n t+t^{3} / 3} \\
\frac{-t^{2}+n}{n t+t^{3} / 3} \\
1
\end{array}\right)
$$

where $t=\frac{X^{1}}{X^{0}}$ and $\widetilde{\mathcal{F}}_{0}$ is the prepotential in the new basis. In these variables, the coupling constant is given by $\tau=\frac{\partial \widetilde{F}_{1}}{\partial \widetilde{X}^{1}}$. Straightforward calculation gives:

$$
\tau=\frac{i}{4 \sqrt{|n|}}
$$

at the critical point (4.11), where $t=i \sqrt{|n|}$. Thus, all the solutions from the infinite family (4.11) have $\operatorname{Im} \tau>0$ and correspond to the maxima of the entropy functional. This simple example illustrates the behavior of a one-parameter Calabi-Yau near the large complex structure limit, $\operatorname{Im} t \gg 1$. In this limit, prepotential receives small instanton corrections and contains subleading quadratic and linear terms. However, in principle one could still find the infinite family of aligned periods by solving appropriately modified equation (4.11):

$$
\operatorname{Re} F_{1}+n X^{0}=0
$$

since the periods $X^{1}$ and $F_{0}$ in general case are imaginary along the zero $B$-field line $\operatorname{Re} X^{1}=0, \operatorname{Im} X^{0}=0$. These will correspond, in the large $\operatorname{Im} t$ limit to small perturbations of the solutions we have already discussed above.

However, we do not get interesting effective field theories at such points, and the relative weight ("probability") of such points is much smaller than that of the maxima where new massless degrees of freedom appear, because of the logarithmic behavior (4.6) of the coupling constant at the singular points. 


\subsection{The Quintic}

Let us consider, following [14], the type IIA superstrings on the well studied compact Calabi-Yau manifold with $h^{2,1}=1$, which is the mirror of the quintic hypersurface in $\mathbf{C P}^{4}$. It can be obtained as a $\left(\mathbb{Z}_{5}\right)^{3}$ quotient of the special quintic

$$
\sum_{i=1}^{5} z_{i}^{5}-5 \psi \prod_{i=1}^{5} z_{i}=0
$$

The complex moduli space is $\mathbf{C} \mathbf{P}^{1}$, parameterized by $z=\psi^{-5}$, with three special points:

$$
\begin{array}{ll}
z=0: & \text { large complex structure limit } \\
z=1: & \text { conifold point } \\
z=\infty: & \text { Gepner point }
\end{array}
$$

The four periods undergo monodromy about these three points. It is convenient to use the basis of the periods corresponding to the BPS state in the mirror A-model labeled by the $(D 6, D 4, D 2, D 0)$-brane charges: $\left(\Pi_{D 0}, \Pi_{D 2}, \Pi_{D 4}, \Pi_{D 6}\right)$. These periods provide corresponding D-brane tensions. The general expression for the prepotential is

$$
F(t)=-\frac{5}{6} t^{3}-\frac{11}{4} t^{2}+\frac{25}{12} t-\frac{25 i}{2 \pi^{3}} \zeta(3)+i \sum_{k=1}^{\infty} \frac{d_{k}}{(2 \pi k)^{3}} e^{2 \pi i k t}
$$

where

$$
t=\frac{\Pi_{D 2}}{\Pi_{D 0}}=\frac{1}{2 \pi i} \log z+\ldots
$$

is a mirror map, and $d_{k}$ are instanton amplitudes (Gromov-Witten invariants), related to the number $n_{k}$ of rational curves of degree $k$ embedded in the quintic, as

$$
\sum_{k=0}^{\infty} d_{k} e^{2 \pi i k t}=5+\sum_{k=1}^{\infty} \frac{n_{k} k^{3}}{1-e^{2 \pi i t}} e^{2 \pi i k t}
$$

We are interested in the solutions to the extremum equations (3.11). As was discussed before, we expect an infinite family of such solutions at zero $B$-field in the large complex structure limit. Indeed, the periods in the basis (3.2)-(3.3) are given by

$$
\left(\begin{array}{c}
X^{0} \\
X^{1} \\
F_{1} \\
F_{0}
\end{array}\right)=\left(\begin{array}{c}
1 \\
t \\
-\frac{5}{2} t^{2}-\frac{11}{2} t+\frac{25}{12}-\sum_{k=1}^{\infty} \frac{d_{k}}{(2 \pi k)^{2}} e^{2 \pi i k t} \\
\frac{5}{6} t^{3}+\frac{25}{12} t-\frac{25 i}{\pi^{3}} \zeta(3)+\sum_{k=1}^{\infty}(2 i+2 \pi k t) \frac{d_{k}}{(2 \pi k)^{3}} e^{2 \pi i k t}
\end{array}\right)
$$


Therefore, at the special set of points, where Ret $=0$ and a deformed CM-type equation holds:

$$
\frac{5}{2} t^{2}-\frac{25}{12}+\sum_{k=1}^{\infty} \frac{d_{k}}{(2 \pi k)^{2}} e^{2 \pi i k t}=n
$$

the following three periods are aligned: $\left(X^{1}, F_{0}, F^{1}+n X^{0}\right)$, where $n$ is an integer. It is clear that when $n \gg 1$ the instanton corrections in (4.22) are small and general behavior is similar to the cubic prepotential case. Therefore, we conclude that this infinite set of solutions describes local maxima of the entropy functional.

As we discussed earlier, the conifold point $z=1$ is a potential candidate for a critical point of the entropy functional. Let us then look at the periods and try to find out which of them are aligned. The periods satisfy corresponding Picard-Fuchs differential equation of hypergeometric type:

$$
\left[\theta_{z}^{4}-\left(\theta_{z}+\frac{1}{5}\right)\left(\theta_{z}+\frac{2}{5}\right)\left(\theta_{z}+\frac{3}{5}\right)\left(\theta_{z}+\frac{4}{5}\right)\right] \Pi_{i}=0
$$

where $\theta_{z}=z \frac{d}{d z}$. We use the conventions of 15 to write down the basis of the solutions to 4.23 as follows:

$$
\begin{aligned}
& \Pi_{0}(z)=U_{0}(z) \\
& \Pi_{1}(z)=U_{1}(z) \text { if } \operatorname{Im} z<0, \quad U_{1}(z)+U_{0}(z) \text { if } \operatorname{Im} z>0 \\
& \Pi_{2}(z)=U_{2}(z) \\
& \Pi_{3}(z)=U_{3}(z) \text { if } \operatorname{Im} z<0, \quad U_{3}(z)+U_{2}(z) \text { if } \operatorname{Im} z>0
\end{aligned}
$$

where $U_{i}$ are given in terms of the Meijer G-function 16]

$$
\begin{aligned}
U_{0}(z) & =c G_{0,3}^{1,4}\left(-z \mid \begin{array}{rrrr}
\frac{4}{5} & \frac{3}{5} & \frac{2}{5} & \frac{1}{5} \\
0 & 0 & 0 & 0
\end{array}\right) \\
U_{1}(z) & =\frac{c}{2 \pi i} G_{1,2}^{2,4}\left(z \mid \begin{array}{rrrr}
\frac{4}{5} & \frac{3}{5} & \frac{2}{5} & \frac{1}{5} \\
0 & 0 & 0 & 0
\end{array}\right) \\
U_{2}(z) & =\frac{c}{(2 \pi i)^{2}} G_{1,1}^{3,4}\left(-z \mid \begin{array}{r|rrrr} 
& \frac{4}{5} & \frac{3}{5} & \frac{2}{5} & \frac{1}{5} \\
0 & 0 & 0 & 0
\end{array}\right) \\
U_{3}(z) & =\frac{c}{(2 \pi i)^{3}} G_{1,0}^{4,4}\left(z \mid \begin{array}{rrrrr} 
& \frac{4}{5} & \frac{3}{5} & \frac{2}{5} & \frac{1}{5} \\
0 & 0 & 0 & 0
\end{array}\right)
\end{aligned}
$$

and

$$
c=\frac{1}{\Gamma\left(\frac{1}{5}\right) \Gamma\left(\frac{2}{5}\right) \Gamma\left(\frac{3}{5}\right) \Gamma\left(\frac{4}{5}\right)}
$$


Near $z=0$ the periods $\Pi_{j}$ behave as $(\log z)^{j}$. One can go to another natural basis, corresponding to $(D 6, D 4, D 2, D 0)$-brane state with the help of the following transformation matrix

$$
\left(\begin{array}{l}
\Pi_{D 6} \\
\Pi_{D 4} \\
\Pi_{D 2} \\
\Pi_{D 0}
\end{array}\right)=\left(\begin{array}{cccc}
0 & 5 & 0 & 5 \\
0 & 1 & -5 & 0 \\
0 & -1 & 0 & 0 \\
1 & 0 & 0 & 0
\end{array}\right)\left(\begin{array}{l}
\Pi_{0} \\
\Pi_{1} \\
\Pi_{2} \\
\Pi_{3}
\end{array}\right)
$$

The intersection form in the new basis is defined by $\#(D 6 \cap D 0)=1$ and $\#(D 2 \cap D 4)=1$.

Straightforward calculation at the conifold point $z=e^{-i 0}$ with the help of the Mathematica package gives:

$$
\left(\begin{array}{l}
\Pi_{D 6} \\
\Pi_{D 4} \\
\Pi_{D 2} \\
\Pi_{D 0}
\end{array}\right)=\left(\begin{array}{c}
0 \\
5 \alpha-7 i \beta \\
2 i \beta \\
\gamma
\end{array}\right)
$$

where $(\alpha, \beta, \gamma)$ are real constants:

$$
\begin{aligned}
\alpha & =-\frac{\sqrt{5}}{16 \pi^{4}} \operatorname{Re} G_{0,1}^{3,4}\left(-1 \mid \begin{array}{rrrrr}
\frac{1}{5} & \frac{2}{5} & \frac{3}{5} & \frac{4}{5} \\
& 0 & 0 & 0 & 0
\end{array}\right) \approx-1.239 \\
\beta & =\frac{\sqrt{5}}{16 \pi^{3}} G_{0,2}^{2,4}\left(1 \mid \begin{array}{rrrr}
\frac{1}{5} & \frac{2}{5} & \frac{3}{5} & \frac{4}{5} \\
0 & 0 & 0 & 0
\end{array}\right) \approx 0.646787 \\
\gamma & ={ }_{4} F_{3}\left(\frac{1}{5}, \frac{2}{5}, \frac{3}{5}, \frac{4}{5} ; 1,1,1 ; 1\right) \approx 1.07073
\end{aligned}
$$

From (4.28) we see that it is possible to align three periods by choosing appropriate linear transformation. For example, we can take

$$
\left(\begin{array}{llll}
2 & 0 & 0 & 0 \\
0 & 2 & 7 & 0 \\
0 & 0 & 1 & 0 \\
0 & 0 & 0 & 1
\end{array}\right)\left(\begin{array}{l}
\Pi_{D 6} \\
\Pi_{D 4} \\
\Pi_{D 2} \\
\Pi_{D 0}
\end{array}\right)=\left(\begin{array}{c}
2 \Pi_{D 6} \\
2 \Pi_{D 4}+7 \Pi_{D 2} \\
\Pi_{D 2} \\
\Pi_{D 0}
\end{array}\right)=\left(\begin{array}{c}
0 \\
10 \gamma \\
2 i \beta \\
\alpha
\end{array}\right)
$$

Therefore, the conifold point in the quintic is a solution to the extremum equations (3.11) if we fix appropriate 3 -cycle. In particular, the choice (4.30) corresponds to fixing $X^{0}=$ $2 \Pi_{D 4}+7 \Pi_{D 2}$.

7 We fix this choice of the branch cut by requiring that D6 brane become massless at the conifold point. 


\subsection{A Multi-parameter Model}

Finally, we consider an example of a non-compact Calabi-Yau manifold with several moduli fields. Such models exhibit some new phenomena. For example, there can be points in the moduli space where several different periods vanish, and the corresponding BPS states become massless. In general, one might expect such points to be saddle points for the entropy functional (neither maxima nor minima). This is indeed what we find in a specific example considered below.

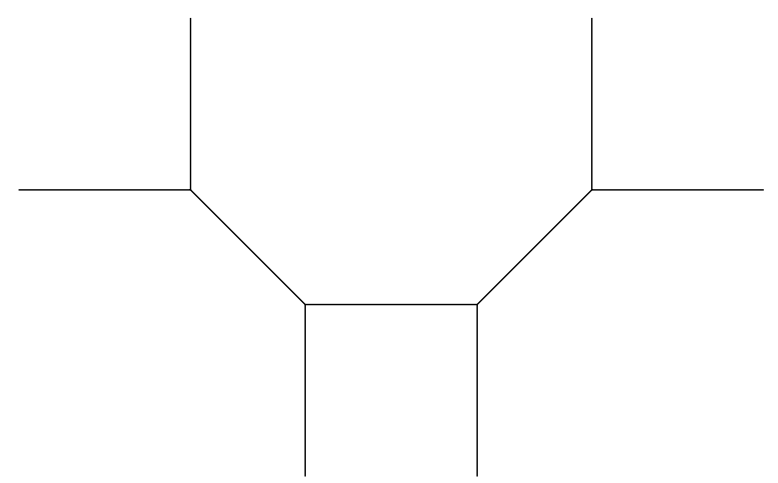

Fig. 2: The toric diagram of the 3-parameter model.

Consider a 3-parameter model studied e.g. in [17]. It has three 2-cycles, whose Kähler parameters we denote by $t_{1}, t_{2}$, and $r$. The prepotential has the form:

$$
F_{\text {top }}^{0}=\sum_{n} \frac{e^{-n t_{1}}}{n^{3}} \sum_{n} \frac{e^{-n t_{2}}}{n^{3}}+\sum_{n} \frac{e^{-n r}\left(1-e^{-n t_{1}}\right)\left(1-e^{-n t_{2}}\right)}{n^{3}}
$$

and the dual variables are

$$
\begin{aligned}
t_{1}^{D} & =\frac{1}{4 \pi^{2}} \sum_{n} \frac{e^{-n t_{1}}\left(1-e^{-n r}\left(1-e^{-n t_{2}}\right)\right)}{n^{2}} \\
t_{2}^{D} & =\frac{1}{4 \pi^{2}} \sum_{n} \frac{e^{-n t_{2}}\left(1-e^{-n r}\left(1-e^{-n t_{1}}\right)\right)}{n^{2}} \\
r^{D} & =\frac{1}{4 \pi^{2}} \sum_{n} \frac{e^{-n r}\left(1-e^{-n t_{1}}\right)\left(1-e^{-n t_{2}}\right)}{n^{2}}
\end{aligned}
$$

8 In notations (3.6), they are given by $t_{1}=-2 \pi i a^{1}, t_{2}=-2 \pi i a^{2}, r=-2 \pi i a^{3}$. 
The coupling constant matrix $\tau_{i j}$ is given by (4.5) and has the following entries (symmetric in $i, j=1,2,3)$ :

$$
\begin{aligned}
& \tau_{11}=-\frac{i}{2 \pi} \log \frac{\left(1-e^{-t_{1}}\right)\left(1-e^{-\left(r+t_{1}+t_{2}\right)}\right)}{\left(1-e^{-\left(r+t_{1}\right)}\right)} \\
& \tau_{22}=-\frac{i}{2 \pi} \log \frac{\left(1-e^{-t_{2}}\right)\left(1-e^{-\left(r+t_{1}+t_{2}\right)}\right)}{\left(1-e^{-\left(r+t_{2}\right)}\right)} \\
& \tau_{12}=-\frac{i}{2 \pi} \log \left(1-e^{-\left(r+t_{1}+t_{2}\right)}\right) \\
& \tau_{13}=-\frac{i}{2 \pi} \log \frac{\left(1-e^{-\left(r+t_{1}+t_{2}\right)}\right)}{\left(1-e^{-\left(r+t_{1}\right)}\right)} \\
& \tau_{23}=-\frac{i}{2 \pi} \log \frac{\left(1-e^{-\left(r+t_{1}+t_{2}\right)}\right)}{\left(1-e^{-\left(r+t_{2}\right)}\right)} \\
& \tau_{33}=-\frac{i}{2 \pi} \log \frac{\left(1-e^{-r}\right)\left(1-e^{-\left(r+t_{1}+t_{2}\right)}\right)}{\left(1-e^{-\left(r+t_{1}\right)}\right)\left(1-e^{-\left(r+t_{2}\right)}\right)}
\end{aligned}
$$

Consider taking the limit $\left(t_{1}, t_{2}, r\right) \rightarrow 0$ along the imaginary line, such that the ratios $t_{1} / r$ and $t_{2} / r$ are kept fixed. Then imaginary parts of the dual variables (4.32) are zero. Therefore, it is a particular solution to the extremum equations (3.17).

In order to determine the behavior of the entropy functional near this extremum, we should diagonalize imaginary part of the matrix $\tau_{i j}$ and look at the eigenvalues. It is easy to see that in this limit it is given by:

$$
\operatorname{Im} \tau=-\frac{\log |r|}{2 \pi}\left(\begin{array}{ccc}
1+\mathcal{O}(x) & 1+\mathcal{O}(x) & \mathcal{O}(x) \\
1+\mathcal{O}(x) & 1+\mathcal{O}(x) & \mathcal{O}(x) \\
\mathcal{O}(x) & \mathcal{O}(x) & -1+\mathcal{O}(x)
\end{array}\right)
$$

where $x \sim \log ^{-1}|r|$. To solve the diagonalization problem to the leading order in $x$, it is enough to consider the matrix of the form

$$
\left(\begin{array}{ccc}
1 & 1 & a \\
1 & 1 & b \\
a & b & -1
\end{array}\right)
$$

where $a \ll 1$ and $b \sim a$. The eigenvalues of this matrix are given by $\left(1, \frac{1}{2}(a-b)^{2},-2\right)$ to the leading order. Therefore, the imaginary part of the coupling matrix (4.34) near the extremum point has one large positive eigenvalue of order $\log |r|$, one positive eigenvalue of order 1 , and one large negative eigenvalue of order $\log |r|$. Notice that $\operatorname{sign}(\operatorname{Im} \tau)=(2,1)$ and therefore, this is an example of a signature of type $\left(h^{2,1}-1,1\right)$. In this case we are having a saddle point of the entropy functional. 


\section{Conclusions and Further Issues}

In this paper we discussed the behavior of the stringy wave function on the moduli space of a Calabi-Yau manifold. It became a meaningful quantity once we fixed a particular combination of charge/chemical potential for one of the magnetic/electric charges of the black hole. The square of this wave function can be interpreted as a measure for string compactifications. As we discussed, the solution to finding maxima/minima of this function has a nice geometric meaning: they correspond to points on the moduli space where all but one period of the holomorphic 3-form $\Omega$ have equal phase. The formulation of this geometric problem involves a choice of a 3 -cycle $A_{0} \in H_{3}(M, \mathbb{Z})$, whose period we denote $X^{0}$ (or, a choice of $A_{0} \in H^{\text {even }}(M, \mathbb{Z})$ in type IIA theory).

While it appears to be a rather challenging problem to obtain a complete solution to these equations, we managed to find a certain class of solutions. They fall into two families: They either correspond to 'quantum deformed' complex multiplication points on the moduli space of a Calabi-Yau manifold, or to points with extra massless particles. Moreover, for the examples with extra massless degrees of freedom the maxima that we found correspond to the points where the effective field theory is asymptotically free.

As discussed above, in order to write down our wave function, we need to choose a particular direction in the charge lattice. In the type IIB case, this corresponds to choosing an integral 3-cycle. It is natural to ask how our conclusions depend on this choice (for the type IIA on non-compact CY there is a natural direction of the charge lattice which corresponds to D0 brane charge). For a compact manifold $M$ there is no natural choice of $A_{0} \in H_{3}(M, \mathbb{Z})$. In fact, even for a particular choice of $A_{0}$, there is an ambiguity related to the monodromy action on $H_{3}(M, \mathbb{Z})$. To classify these choices we need to study the monodromy group action on $H_{3}(M, \mathbb{Z})$. For a Calabi-Yau manifold $M$, the monodromy group $H$ is a subgroup of $G=S p\left(2 h^{2,1}+2, \mathbb{Z}\right)$, so that the number of distinct choices of a 3-cycle is given by the index $[G: H]$. The calculation of $[G: H]$ for a compact CalabiYau space is an interesting and challenging problem. By analogy with the mapping class

group of a genus- $g$ Riemann surface [18], we may expect that $[G: H]$ is finite. In fact, the monodromy group $H$ can be generated by two elements, which correspond to monodromies around the conifold point and infinity. For example, for the quintic threefold, we have

$$
M_{c}=\left(\begin{array}{cccc}
1 & 0 & 0 & 0 \\
0 & 1 & 0 & 0 \\
-1 & 0 & 1 & 0 \\
0 & 0 & 0 & 1
\end{array}\right) \quad M_{\infty}=\left(\begin{array}{cccc}
1 & -1 & 5 & -3 \\
0 & 1 & -8 & -5 \\
0 & 0 & 1 & 0 \\
0 & 0 & 1 & 1
\end{array}\right)
$$


In this case, it is easy to see that $[G: H]>1$.

Another important question that deserves further study is classification of the extremum points on the moduli space, as solutions to the equations (3.17). In mathematical terms, the problem is to find all the points on the moduli space where all but one of the Calabi-Yau periods are aligned.

Finally it would be interesting to understand more physically what it means to fix a charge/chemical potential, and why that is natural. It is conceivable that this becomes natural in the context of decoupling gravity from gauge theory. In particular a preferred direction may be the direction corresponding to the graviphoton charge. It is worthwhile trying to dynamically explain such a formulation of the problem.

\section{Acknowledgments}

We would like to thank R. Dijkgraaf, S. Katz, A. Klemm, M. Roček, T. Oliker and H. Ooguri for valuable discussions. We also thank B.Fiol for pointing out a sign error in the large complex structure limit example, that appeared in the first version of the paper.

This research was supported in part by NSF grants PHY-0244821 and DMS-0244464. This work was conducted during the period S.G. served as a Clay Mathematics Institute Long-Term Prize Fellow. K.S. and S.G. are also supported in part by RFBR grant 04-0216880. We would like to thank the 2005 Simons Workshop on Mathematics and Physics

for providing a stimulating environment where part of this work was done. S.G. would also like to thank the KITP at Santa Barbara for hospitality during the completion of this work. While at KITP, the research of S.G. was supported in part by by the NSF under grant PHY99-07949. 


\section{References}

[1] S. Kachru, R. Kallosh, A. Linde and S. P. Trivedi, "De Sitter vacua in string theory," Phys. Rev. D 68, 046005 (2003) arXiv:hep-th/0301240.

[2] M. R. Douglas, "Basic results in vacuum statistics," Comptes Rendus Physique 5, 965 (2004) arXiv:hep-th/0409207.

[3] H. Ooguri, C. Vafa and E. Verlinde, "Hartle-Hawking wave-function for flux compactifications," hep-th/0502211.

[4] H. Ooguri, A. Strominger and C. Vafa, "Black hole attractors and the topological string," Phys. Rev. D 70, 106007 (2004) arXiv:hep-th/0405146.

[5] S. Ferrara, R. Kallosh and A. Strominger, "N=2 extremal black holes," Phys. Rev. D 52, 5412 (1995).

[6] P. Candelas and X. de la Ossa, "Moduli Space Of Calabi-Yau Manifolds," Nucl. Phys. B 355, 455 (1991).

[7] D. Mumford, "A note on Shimura's paper Discontinuous Groups and Abelian Varieties," Math. Ann. 181 (1969) 345.

[8] C. Borcea, "Calabi-Yau Threefolds and Complex Multiplication," in Essays on Mirror Manifolds, S.-T. Yau ed., International Press, 1992.

[9] G. Moore, "Arithmetic and attractors," arXiv:hep-th/9807087.

[10] S. Gukov and C. Vafa, "Rational conformal field theories and complex multiplication," Commun. Math. Phys. 246, 181 (2004), hep-th/0203213.

[11] E. Cremmer, C. Kounnas, A. Van Proeyen, J. P. Derendinger, S. Ferrara, B. de Wit and L. Girardello, "Vector Multiplets Coupled To N=2 Supergravity: Superhiggs Effect, Flat Potentials And Geometric Structure," Nucl. Phys. B 250, 385 (1985).

[12] S. Gukov, K. Saraikin and C. Vafa, "A stringy wave function for an $S^{3}$ cosmology," hep-th/0505204.

[13] R. Gopakumar and C. Vafa, "M-theory and topological strings. I, II" arXiv:hepth/9809187, arXiv:hep-th/9812127.

[14] P. Candelas, X. C. De La Ossa, P. S. Green and L. Parkes, "A Pair Of Calabi-Yau Manifolds As An Exactly Soluble Superconformal Theory," Nucl. Phys. B 359, 21 (1991).

[15] F. Denef, B. R. Greene and M. Raugas, "Split attractor flows and the spectrum of BPS D-branes on the quintic," JHEP 0105, 012 (2001)

[16] The Bateman Project, Higher Transcendental Functions, Vol. 1, Sec. 5.3.-5.6, A. Erdelyi ed., McGraw-Hill Book Company, New York (1953).

[17] M. Aganagic, M. Marino and C. Vafa, "All loop topological string amplitudes from Chern-Simons theory," Commun. Math. Phys. 247 (2004) 467, hep-th/0206164.

[18] B. Wajnryb, "Mapping class group of a surface is generated by two elements," Topology 35 (1996) 377. 